\title{
Reviewers for this Special Issue
}

We would like to thank the following people who reviewed papers submitted for publication in this special issue: The First 20 Years of Law and Human Behavior.

Donald Bersoff

Bette Bottoms

John Bringham

Stephen Ceci

David N. Cox

Shari Diamond

Kevin S. Douglas

David Faigman

Norman Finkel

Adelle Forth

Solomon Fulero

Jane Goodman-Delahunty

Alexander Greer

Barbara Gutek

Craig Haney

Grant Harris

Stephen D. Hart

Kirk Heilbrun
Steven Hoge

Margaret Bull Kovera

James Luginbuhl

Gary Moran

Michael Nietzel

Randy K. Otto

Jeffrey E. Pfeifer

Norman Poythress

V. Gordon Rose

Michael Saks

Ralph Serin

Christopher Slobogin

Alan Tomkins

Melissa Warren

Stephen Wong

Steven Wormith

Daniel Yarmey

John Yuille 